\title{
Hanseníase no contexto da Estratégia Saúde da Família em cenário endêmico do Maranhão: prevalência e fatores associados
}

\author{
Leprosy in the context of the Family Health Strategy in an endemic \\ scenario in Maranhão: prevalence and associated factors
}

Fernanda de Castro Lopes (https://orcid.org/0000-0003-4578-792X)1 ${ }^{1}$

Antônio Carlos Vieira Ramos (https://orcid.org/0000-0002-7862-1355) ${ }^{2}$

Lívia Maia Pascoal (https://orcid.org/0000-0003-0876-3996) (1,3 $^{1,0}$

Floriacy Stabnow Santos (https://orcid.org/0000-0001-7840-7642) ${ }^{3}$

Isaura Leticia Tavares Palmeira Rolim (https://orcid.org/0000-0002-8453-2543) ${ }^{1}$

Maria Aparecida Alves de Oliveira Serra (https://orcid.org/0000-0003-0952-9560) ${ }^{3}$

Leonardo Hunaldo dos Santos (https://orcid.org/0000-0003-2280-4643) ${ }^{3}$

Marcelino Santos Neto (https://orcid.org/0000-0002-6105-1886) 1,3 $^{1,3}$

\footnotetext{
${ }^{1}$ Programa de PósGraduação em Enfermagem, Universidade Federal do Maranhão. Av. dos Portugueses 1966, Vila Bacanga. 65080-805 São Luís MA Brasil. marcelinosn@gmail.com ${ }^{2}$ Escola de Enfermagem de Ribeirão Preto, Universidade de São Paulo. Ribeirão Preto SP Brasil.

${ }^{3}$ Programa de PósGraduação em Saúde e Tecnologia, Universidade Federal do Maranhão. Imperatriz MA Brasil.
}

\begin{abstract}
This study aimed to describe the prevalence of leprosy and verify the factors associated with multibacillary clinical types in a Family Health Strategy priority setting to control and monitor the disease in northeastern Brazil. This cross-sectional study used data from the Notifiable Diseases Information System related to leprosy cases notified in Imperatriz, Maranhão, between 2008 and 2017. The prevalence was determined yearly and for the period. Poisson regression models with a significance level of 5\% were employed to associate the variables and the multibacillary clinical types. Most of the 2,476 leprosy cases analyzed referred to multibacillary clinical types. The prevalence ranged from 7.8 and 15.6/10,000 inhabitants, with high and very high endemicity levels. The variables male, age groups between 30 and 59 years and $\geq 60$ years, schooling $<8$ years, level 2 physical disability, types 1 and 2 reactive episodes, and urban residence area showed significant associations $(p \leq 0.05)$ with multibacillary clinical types. Such findings can serve as a basis for elaborating and implementing leprosy control and surveillance measures, gearing actions to the most vulnerable groups, and becoming more effective. Keywords Leprosy, Epidemiology, Family Health Strategy
\end{abstract}

Resumo Este estudo objetivou descrever a prevalência da hanseníase e verificar os fatores associados às formas clínicas multibacilares em cenário da Estratégia Saúde da Família, prioritário para o controle e a vigilância da doença no nordeste brasileiro. Trata-se de estudo transversal, que utilizou dados do Sistema de Informação de Agravos de Notificação relativos aos casos de hanseníase notificados em Imperatriz, Maranhão, entre 2008 e 2017. Determinaram-se as prevalências a cada ano e para o período. Para associar as variáveis e as formas clínicas multibacilares, utilizaram-se modelos de regressão de Poisson, com nível de significância de 5\%. Dos 2.476 casos de hanseníase analisados, a maioria referiu-se às formas clínicas multibacilares. A prevalência variou entre 15,6 e 7,8/10 mil habitantes, encontrando-se níveis alto e muito alto de endemicidade. As variáveis sexo masculino, faixas etárias entre 30 e 59 anos e $\geq 60$ anos, escolaridade $<8$ anos, grau 2 de incapacidade física, episódio reacional tipos 1 e 2 e zona de residência urbana apresentaram associações significativas $(p \leq 0,05)$ com as formas clinicas multibacilares. Tais achados podem servir de base para elaboração e implementação de medidas de controle e vigilância da hanseníase, direcionando as ações para os grupos mais vulneráveis e tornando-se mais efetivas.

Palavras-chave Hanseníase, Epidemiologia, Estratégia Saúde da Família 


\section{Introdução}

Considerada uma das doenças mais antigas que acomete o homem, a hanseníase está associada à desigualdade social e a condições socioeconômicas desfavoráveis, como analfabetismo, condições precárias de moradia, falta de saneamento básico, crescimento urbano desorganizado e serviços de saúde ineficazes ${ }^{1-3}$.

Apesar de ter sido eliminada como problema de saúde pública em muitos países (prevalência $<1$ caso a cada 10 mil habitantes) desde o ano 2000, a hanseníase ainda persiste naqueles em desenvolvimento como uma doença negligencia$\mathrm{da}^{4-6}$, capaz de ocasionar limitações físicas e sociais a seus portadores e aumentar os custos para os serviços de saúde, além de contribuir para a estagnação do panorama de desigualdades sociais dessas localidades ${ }^{5,6}$.

Dados da Organização Mundial da Saúde (OMS) revelaram que, em 2018, 208.619 novos casos da doença foram detectados globalmente, distribuídos em 161 países, com taxa de detecção de 2,74 casos por 100 mil habitantes e prevalência de 0,29 caso por 10 mil habitantes ${ }^{7}$. Em comparação ao ano anterior, a taxa global de prevalência teve decréscimo de $4 \%$, mas países das Américas, do Mediterrâneo e do Pacífico Ocidental apresentaram aumentos nas taxas, chegando a 0,58 caso por 10 mil habitantes 7 .

O Brasil mantém-se na lista dos 23 países prioritários para o controle da doença e, em conjunto com a Îndia e a Indonésia, foi responsável por cerca de $80 \%$ dos casos novos registrados em $2018^{7,8}$. Ademais, permanece ocupando o primeiro lugar das Américas, contribuindo com 93\% dos casos novos registrados em 2018, com prevalência de 1,48 casos por 10 mil habitantes ${ }^{7,8}$. Nos últimos 5 anos, a maior concentração dos casos ocorreu nas Regiões Norte, Nordeste e Centro -Oeste do país.

Em se tratando das unidades federativas endêmicas, destaca-se o Maranhão (MA), na Região Nordeste do Brasil, que apresentou, entre 2014 e 2018, taxa média de detecção de casos novos de 79,7 por 100 mil habitantes e prevalência média de 4,33 casos por 10 mil habitantes ${ }^{8}$. Entre os municípios maranhenses, a cidade de Imperatriz, no final do ano de 2017, apresentou taxa de incidência de 73,87 por 100 mil habitantes", sendo considerada prioritária para o controle e a vigilância da doença.

Em decorrência da magnitude da hanseníase como problema de saúde pública brasileira, o Programa Nacional de Controle da Hanseníase do Ministério da Saúde preconizou que as ações de controle da doença fossem descentralizadas para a Atenção Primária à Saúde (APS) e coordenadas pela Estratégia Saúde da Família (ESF), a fim de facilitar o acesso da população aos serviços, garantir a qualidade da assistência e reduzir os níveis endêmicos da doença ${ }^{10}$. Apesar dessa normativa, a prática clínica e as investigações científicas continuam suscitando dificuldades para a realização do diagnóstico precoce e tratamento oportuno da doença, da prevenção e do monitoramento das incapacidades físicas, além da vigilância dos contatos, o que tem acarretado sequelas motoras e neurológicas na população acometida $^{11}$.

De acordo com a OMS, para fins operacionais de tratamento, os doentes são classificados em paucibacilares $(\mathrm{PB})$, com presença de até cinco lesões de pele e baciloscopia de raspado intradérmico negativo, quando disponível, ou multibacilares (MB), com presença de seis ou mais lesões de pele ou baciloscopia de raspado intradérmico positiva ${ }^{1,4}$. A classificação MB sobreleva-se por representar as formas mais graves da doença, diretamente relacionadas à maior transmissibilidade de Mycobacterium leprae, até que seja iniciado o tratamento com a poliquimioterapia $(\mathrm{PQT})^{12}$.

Destaca-se ainda que, no Brasil, ocorreu aumento na proporção de $\mathrm{MB}$ entre o total de casos novos. Em 2009, a proporção foi de $57,2 \%$ e, em 2018, apresentou proporção de 77,2\%. Esse incremento foi notabilizado em todas as regiões do país, assim como no estado do Maranhão, que apresentou proporção de $80,8 \%{ }^{8}$.

Assim, evidencia-se a necessidade de conhecer e analisar aspectos epidemiológicos dos casos notificados de hanseníase, no que diz respeito à totalidade dos casos em tratamento em localidades prioritárias para o monitoramento da doença, com vistas à compreensão de fatores associados às formas clínicas com maior possibilidade de propagação da doença, fornecendo subsídios para planejamento e intervenções de saúde mais efetivas.

Desse modo, objetivou-se determinar a prevalência da hanseníase e verificar os fatores associados às formas clínicas MB.

\section{Métodos}

Trata-se de estudo transversal ${ }^{13}$, realizado em Imperatriz (MA), que possui área territorial de $1.368,988 \mathrm{~km}^{2}$ e população estimada em 258.016 
habitantes no ano de 2018, sendo mais de $94 \%$ destes residentes na área urbana ${ }^{14}$. O município está localizado a $626 \mathrm{~km}$ da capital do estado, São Luís, e é considerado o segundo maior centro populacional, comercial e de serviços do Maranhão ${ }^{14,15}$.

Foram incluídos todos os casos de hanseníase notificados junto ao Sistema de Informação de Agravos de Notificação (Sinan) no período compreendido entre janeiro de 2008 e dezembro de 2017, coletados em fevereiro de 2019 no Serviço de Vigilância em Saúde (SVS) da Unidade Gestora Regional de Saúde de Imperatriz. Após a coleta de dados, foram excluídos registros em duplicidade ( $74 ; 2,90 \%)$ e com situação de encerramento "erro de diagnóstico" $(8 ; 0,31 \%)$.

Foram selecionadas para investigação variáveis sociodemográficas, como idade, sexo, raça/ cor e escolaridade, além de variáveis clínico-epidemiológicas, como modo de entrada, classificação operacional, forma clínica, número de nervos afetados, incapacidade física no momento do diagnóstico, número de contatos registrados, número de contatos examinados, zona de residência e situação de encerramento.

Realizou-se a análise descritiva dos dados, por meio da determinação de frequências absolutas e relativas das variáveis sociodemográficas e clínico-epidemiológicas, segundo a classificação operacional $(\mathrm{MB} / \mathrm{PB})$. Para a variável idade, foram calculados média, mediana, moda e desvio -padrão. A variável idade também foi categorizada em quatro intervalos ( $<15$ anos, 15 a 29 anos, 30 a 59 anos e $\geq 60$ anos).

Determinou-se a taxa de prevalência a cada 10 mil habitantes, para cada ano sob investigação, levando-se em consideração todos os casos em curso de tratamento em 31 de dezembro do ano de avaliação, divididos pela população total do mesmo local e período ${ }^{16}$, de acordo com as estimativas populacionais do Instituto Brasileiro de Geografia e Estatística (IBGE) ${ }^{14}$.

Foram utilizados os parâmetros de endemicidade para a classificação dos achados da prevalência, conforme expresso no item Indicadores de Monitoramento do Progresso da Eliminação da Hanseníase enquanto Problema de Saúde Pública das Diretrizes para Vigilância, Atenção e Eliminação da Hanseníase como Problema de Saúde Pública ${ }^{16}$, sendo consideradas como de baixa endemicidade as localidades com menos de 1,0 caso por 10 mil habitantes; média entre 1,0 a 4,9 casos; alta entre 5,0 e 9,9 casos; muito alta entre 10,0 e 19,9 casos e hiperendêmica $\geq 20,0$ casos por 10 mil habitantes.
Para verificar os fatores associados às formas clínicas MB, foram utilizados modelos de regressão de Poisson com ajuste robusto da variância. As variáveis sociodemográficas e clínico-epidemiológicas que apresentaram valor de $p \leq 0,20$ foram incluídas no modelo ajustado. As razões de prevalência (RP) e os respectivos intervalos de confiança de $95 \%$ foram estimados diretamente pelos coeficientes de regressão do modelo ${ }^{17}$. Nessa ocasião, os dados ignorados foram excluídos. Os dados foram tabulados em planilhas do Microsoft Office Excel ${ }^{\circledR} 2019$ e os testes realizados no programa IBM Statistical Package for Social Science $^{\circledR}$ (SPSS ${ }^{\circledR}$ ), versão 24.0 , a $5 \%$ de significância.

A pesquisa foi aprovada pelo Comitê de Ética em Pesquisa da Universidade Federal do Maranhão (UFMA), de acordo com as diretrizes e normas regulamentadoras de pesquisa em seres humanos das resoluções 466/2012 e 510/2016.

\section{Resultados}

No período sob investigação, foram notificados, junto ao Sinan do município de Imperatriz, 2.550 casos de hanseníase e, após a aplicação dos critérios de exclusão (duplicidade e erro de diagnóstico), foram considerados 2.468 casos para a investigação. Destes, 1.662 (67,34\%) foram classificados como MB e 806 (32,66\%) como PB.

Ao longo do período estudado, a prevalência variou entre 7,8 e 15,6 a cada 10 mil habitantes. Houve diminuição da prevalência aos longos dos anos até 2014, e, após, ela se manteve com taxas semelhantes até o final do período analisado ( $\mathrm{Fi}$ gura 1).

A idade mínima dos casos foi de 3 anos, a máxima foi 97, e a média foi de 41,3 anos, com desvio-padrão de 19,2, mediana de 41 e moda de 28 anos. Ao comparar as características sociodemográficas segundo classificação operacional, observou-se que houve predomínio dos casos $\mathrm{MB}$ em indivíduos do sexo masculino (76,43\%), faixa etária $\geq 60$ anos $(77,52 \%)$, raça/cor parda $(69,54 \%)$, escolaridade $<8$ anos $(72,58 \%)$, além de percentuais expressivos de informações ignoradas em variáveis como raça/cor $(68,42 \%)$ e escolaridade $(68,82 \%)$ (Tabela 1$)$.

Quanto às caraterísticas clínico-epidemiológicas dos casos $\mathrm{MB}$ comparados ao $\mathrm{PB}$, houve predominância de outros reingressos $(90,90 \%)$ como modo de entrada, forma clínica dimorfa $(70,70 \%)$, entre dois e cinco nervos afetados $(67,96 \%)$, grau 2 de incapacidade física $(91,36 \%)$, 


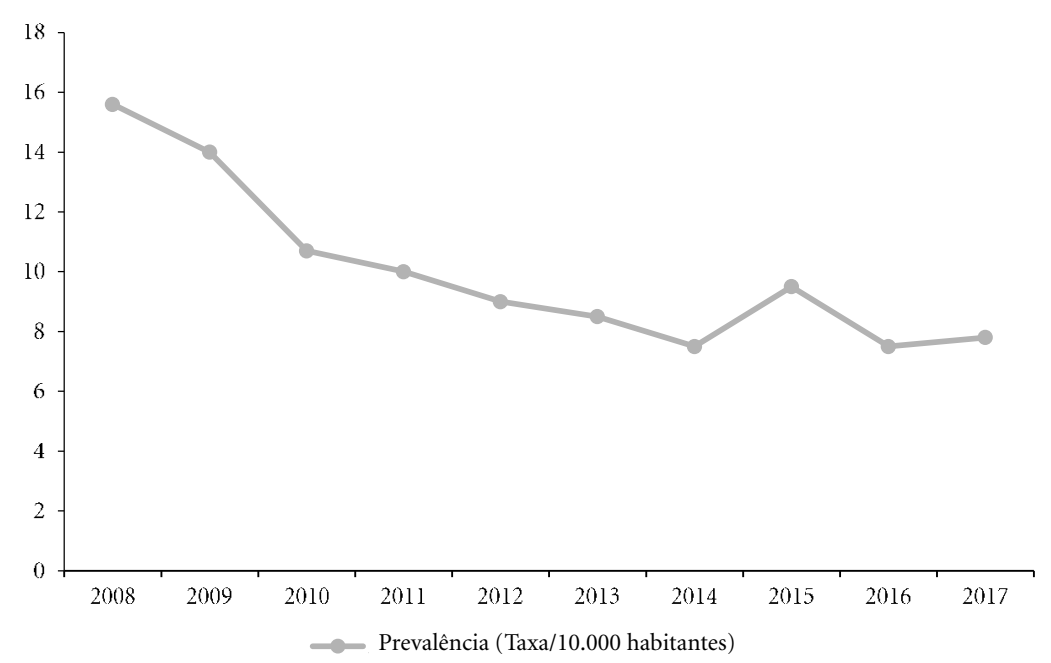

Figura 1. Prevalência de hanseníase no município de Imperatriz, Maranhão, Brasil, 2008 a 2017.

Fonte: SINAN Hanseníase, SVS-UGRSI, Imperatriz-Maranhão, 2019.

Tabela 1. Características sociodemográficas dos casos notificados de hanseníase, segundo classificação operacional, Imperatriz, Maranhão, Brasil, 2008 a 2017.

\begin{tabular}{|c|c|c|}
\hline \multirow[b]{2}{*}{ Variáveis } & \multicolumn{2}{|c|}{$\begin{array}{c}\text { Classificação operacional } \\
(\mathrm{n}=2.468)\end{array}$} \\
\hline & $\begin{array}{c}\text { Paucibacila- } \\
\text { res }(\mathrm{n}=806) \\
\mathrm{n}(\%)\end{array}$ & $\begin{array}{c}\text { Multibacila- } \\
\text { res }(\mathrm{n}=1.662) \\
\mathrm{n}(\%)\end{array}$ \\
\hline \multicolumn{3}{|l|}{ Sexo } \\
\hline Feminino & $463(45,70)$ & $550(54,30)$ \\
\hline Masculino & $343(23,57)$ & $1.112(76,43)$ \\
\hline \multicolumn{3}{|l|}{ Idade, anos } \\
\hline$<15$ & $98(47,53)$ & $108(52,47)$ \\
\hline $15-29$ & $194(35,02)$ & $360(64,98)$ \\
\hline $30-59$ & $409(32,90)$ & $834(67,10)$ \\
\hline$\geq 60$ & $105(22,48)$ & $362(77,52)$ \\
\hline \multicolumn{3}{|l|}{ Raça/cor } \\
\hline Branca & $228(38,00)$ & $372(62,00)$ \\
\hline Preta & $114(30,48)$ & $260(69,52)$ \\
\hline Amarela & $20(39,22)$ & $31(60,78)$ \\
\hline Parda & $427(30,46)$ & $975(69,54)$ \\
\hline Indígena & $8(42,11)$ & $11(57,89)$ \\
\hline Ignorado & $6(31,58)$ & $13(68,42)$ \\
\hline \multicolumn{3}{|c|}{ Escolaridade, anos } \\
\hline$\geq 8$ & $335(41,67)$ & $469(58,33)$ \\
\hline$<8$ & $403(27,41)$ & $1.067(72,58)$ \\
\hline Não se aplica & $17(68,00)$ & $8(32,00)$ \\
\hline Ignorado & $51(30,18)$ & $118(68,82)$ \\
\hline
\end{tabular}

Fonte: SINAN Hanseníase, SVS-UGRSI, Imperatriz-Maranhão, 2019. episódio reacional tipo 2 (93,33\%), com número de contatos registrados e examinados maior que cinco $(67,36 \%$ e $72,28 \%$, respectivamente), residentes em área urbana $(67,59 \%)$ e encerramento por cura $(68,07 \%)$. Observaram-se, ainda, valores percentuais majoritários de informações ignoradas nos registros dos casos $\mathrm{MB}$, especificamente em variáveis como modo de entrada $(82,35 \%)$, nervos afetados $(67,30 \%)$, incapacidade física $(74,07 \%)$, episódio reacional $(64,34 \%)$, número de contatos registrados $(75,00 \%)$, número de contatos examinados $(71,09 \%)$ e tipo de saída $(67,74 \%)$ (Tabela 2).

$\mathrm{Na}$ análise bruta, as associações significativas $(\mathrm{p} \leq 0,20)$ para a classificação operacional MB foram faixas etárias entre 30 e 59 anos e $\geq 60$ anos, sexo masculino, raça/cor parda, escolaridade $<8$ anos, graus 1 e 2 de incapacidade física, episódios reacionais tipos 1 e 2 e zona de residência urbana. $\mathrm{Na}$ análise ajustada, as associações significativas $(\mathrm{p} \leq 0,05)$ para a forma MB foram sexo masculino, faixas etárias entre 30 e 59 anos e $\geq 60$ anos, escolaridade $<8$ anos, grau 2 de incapacidade física, episódios reacionais tipos 1 e 2 e zona de residência urbana (Tabela 3 ). 
Tabela 2. Características clínico-epidemiológicas dos casos notificados de hanseníase segundo classificação operacional, Imperatriz, Maranhão, Brasil, 2008 a 2017.

\begin{tabular}{|c|c|c|}
\hline \multirow[b]{2}{*}{ Variáveis } & \multicolumn{2}{|c|}{$\begin{array}{c}\text { Classificação operacional } \\
(n=2.468)\end{array}$} \\
\hline & $\begin{array}{c}\text { Paucibacilares } \\
(\mathbf{n}=806) \\
\mathrm{n}(\%)\end{array}$ & $\begin{array}{c}\text { Multibacilares } \\
\begin{array}{c}(\mathrm{n}=1.662) \\
\mathrm{n}(\%)\end{array}\end{array}$ \\
\hline \multicolumn{3}{|l|}{ Modo de entrada } \\
\hline Caso novo & $755(37,17)$ & $1.276(62,83)$ \\
\hline Transferências & $13(11,40)$ & $101(88,60)$ \\
\hline Recidiva & $17(20,73)$ & $65(79,27)$ \\
\hline $\begin{array}{l}\text { Outros } \\
\text { reingressos }\end{array}$ & $22(9,10)$ & $220(90,90)$ \\
\hline Ignorado & $6(17,65)$ & $28(82,35)$ \\
\hline \multicolumn{3}{|l|}{ Forma clínica } \\
\hline Indeterminada & $337(41,81)$ & - \\
\hline Tuberculoide & $469(58,19)$ & - \\
\hline Dimorfa & - & $1.175(70,70)$ \\
\hline Virchowiana & - & $487(29,30)$ \\
\hline \multicolumn{3}{|l|}{$\begin{array}{l}\text { Número de } \\
\text { nervos afetados }\end{array}$} \\
\hline 0 & $312(34,21)$ & $600(65,79)$ \\
\hline 1 & $75(32,47)$ & $156(67,53)$ \\
\hline $2-5$ & $116(32,04)$ & $246(67,96)$ \\
\hline$>5$ & $38(32,76)$ & $78(67,24)$ \\
\hline Ignorado & $277(32,70)$ & $570(67,30)$ \\
\hline \multicolumn{3}{|l|}{$\begin{array}{l}\text { Incapacidade } \\
\text { física }\end{array}$} \\
\hline Grau zero & $654(40,55)$ & $959(59,45)$ \\
\hline Grau 1 & $57(13,77)$ & $357(86,23)$ \\
\hline Grau 2 & $14(8,64)$ & $148(91,36)$ \\
\hline Não avaliado & $74(29,36)$ & $178(70,63)$ \\
\hline Ignorado & $7(25,93)$ & $20(74,07)$ \\
\hline
\end{tabular}

\section{Discussão}

Observou-se redução progressiva da prevalência de hanseníase ao longo do período analisado, com valor máximo de 15,6 por 10 mil habitantes, em 2008, e mínimo de 7,8 por 10 mil habitantes, em 2016, sendo o município enquadrado em níveis alto e muito alto de endemicidade ${ }^{16} \mathrm{e}$ com taxas acima da meta de eliminação estabelecida pela $\mathrm{OMS}^{18}$. Tais achados suscitam a premência de análises periódicas dos indicadores epidemio-
Tabela 2. Características clínico-epidemiológicas dos casos notificados de hanseníase segundo classificação operacional, Imperatriz, Maranhão, Brasil, 2008 a 2017.

\begin{tabular}{|c|c|c|}
\hline \multirow[b]{2}{*}{ Variáveis } & \multicolumn{2}{|c|}{$\begin{array}{c}\text { Classificação operacional } \\
(n=2.468)\end{array}$} \\
\hline & $\begin{array}{l}\text { Paucibacilares } \\
\qquad \begin{array}{c}(\mathbf{n}=806) \\
\mathbf{n}(\%)\end{array}\end{array}$ & $\begin{array}{l}\text { Multibacilares } \\
\begin{array}{c}(\mathrm{n}=1.662) \\
\mathrm{n}(\%)\end{array}\end{array}$ \\
\hline \multicolumn{3}{|l|}{ Episódio reacional } \\
\hline Sem reação & $178(29,32)$ & $429(70,68)$ \\
\hline Reação tipo 1 & $11(10,28)$ & $96(89,72)$ \\
\hline Reação tipo 2 & $1(6,67)$ & $14(93,33)$ \\
\hline Reações tipos & $3(15,00)$ & $17(85,00)$ \\
\hline 1 e 2 & & \\
\hline Ignorado & $613(35,66)$ & $1.106(64,34)$ \\
\hline \multicolumn{3}{|l|}{$\begin{array}{l}\text { Número } \\
\text { de contatos } \\
\text { registrados }\end{array}$} \\
\hline$\leq 5$ & $692(32,72)$ & $1.423(67,28)$ \\
\hline$>5$ & $110(32,64)$ & $227(67,36)$ \\
\hline Ignorado & $4(25,00)$ & $12(75,00)$ \\
\hline \multicolumn{3}{|l|}{$\begin{array}{l}\text { Número } \\
\text { de contatos } \\
\text { examinados }\end{array}$} \\
\hline$\leq 5$ & $678(33,61)$ & $1339(66,39)$ \\
\hline$>5$ & $56(27,72)$ & $146(72,28)$ \\
\hline Ignorado & $72(28,91)$ & $177(71,09)$ \\
\hline \multicolumn{3}{|l|}{$\begin{array}{l}\text { Zona de } \\
\text { residência }\end{array}$} \\
\hline Rural & $30(40,54)$ & $44(59,46)$ \\
\hline Urbana & $776(32,41)$ & $1.618(67,59)$ \\
\hline \multicolumn{3}{|l|}{ Tipo de saída } \\
\hline Cura & $636(31,93)$ & $1.356(68,07)$ \\
\hline Transferências & $74(37,00)$ & $126(63,00)$ \\
\hline Óbito & $5(38,46)$ & $8(61,53)$ \\
\hline Abandono & $51(36,70)$ & $88(63,30)$ \\
\hline Ignorado & $40(32,26)$ & $84(67,74)$ \\
\hline
\end{tabular}

Fonte: SINAN Hanseníase, SVS-UGRSI, Imperatriz-Maranhão, 2019.

lógicos e operacionais, para avaliar a efetividade das medidas de controle e a progressão da doen$\mathrm{ça}^{19}$.

O coeficiente de prevalência da hanseníase é considerado indicador essencial para subsidiar a formulação de ações e estratégias de controle da doença, com vistas ao tratamento oportuno dos doentes, à descontinuidade da cadeia de transmissão e à prevenção de incapacidades física ${ }^{20}$.

Estudo conduzido por Ribeiro et al. ${ }^{20}$ revelou tendência nacional similar de diminuição 
Tabela 3. Análise bruta e ajustada das variáveis sociodemográficas e clínico-epidemiológicas associadas às formas clínicas multibacilares, Imperatriz, Maranhão, Brasil, 2008 a 2017.

\begin{tabular}{|c|c|c|c|c|}
\hline \multirow[b]{2}{*}{ Variáveis } & \multicolumn{4}{|c|}{ Classificação operacional multibacilares } \\
\hline & $\begin{array}{l}\text { RP bruta* } \\
\text { (IC95\%) }\end{array}$ & Valor de $p$ & $\begin{array}{c}\text { RP ajustada } \\
(\text { IC95\%) }\end{array}$ & Valor de $\mathrm{p}$ \\
\hline \multicolumn{5}{|l|}{ Idade, anos } \\
\hline$<15$ & 1 & & 1 & \\
\hline $15-29$ & $1,24(1,08-1,43)$ & 0,083 & $1,17(1,00-1,51)$ & 0,091 \\
\hline $30-59$ & $1,28(1,21-1,46)$ & $<0,001$ & $1,60(1,48-1,81)$ & $<0,001$ \\
\hline$\geq 60$ & $1,48(1,29-1,70)$ & $<0,001$ & $1,72(1,51-1,99)$ & $<0,001$ \\
\hline \multicolumn{5}{|l|}{ Sexo } \\
\hline Feminino & 1 & & 1 & \\
\hline Masculino & $1,82(1,73-1,91)$ & $<0,001$ & $1,97(1,89-2,07)$ & $<0,001$ \\
\hline \multicolumn{5}{|l|}{ Raça } \\
\hline Branca & 1 & & - & - \\
\hline Preta & $1,12(1,02-1,23)$ & 0,110 & - & - \\
\hline Amarela & $0,90(0,78-1,24)$ & 0,899 & - & - \\
\hline Parda & $2,12(2,04-2,26)$ & 0,001 & - & - \\
\hline Indígena & $0,94(0,63-1,38)$ & 0,749 & - & - \\
\hline \multicolumn{5}{|l|}{ Escolaridade, anos } \\
\hline$\geq 8$ & 1 & & 1 & \\
\hline$<8$ & $2,24(2,12-2,36)$ & $<0,001$ & $2,50(2,37-2,71)$ & $<0,001$ \\
\hline Não se aplica & $1,05(0,63-1,75)$ & 0,101 & $1,15(0,75-1,88)$ & 0,151 \\
\hline \multicolumn{5}{|l|}{ Modo de entrada } \\
\hline Outros reingressos & 1 & & - & - \\
\hline Caso novo & $0,95(0,88-1,03)$ & 0,100 & - & - \\
\hline Transferências & $0,87(0,77-0,98)$ & 0,228 & - & - \\
\hline Recidiva & $0,69(0,64-0,76)$ & 0,257 & - & - \\
\hline \multicolumn{5}{|c|}{ Número de nervos afetados } \\
\hline Zero & 1 & & - & - \\
\hline 1 & $1,02(0,92-1,13)$ & 0,612 & - & - \\
\hline $2-5$ & $1,03(0,95-1,15)$ & 0,454 & - & - \\
\hline$>5$ & $1,01(0,89-1,16)$ & 0,752 & - & - \\
\hline \multicolumn{5}{|l|}{ Incapacidade física } \\
\hline Grau zero & 1 & & 1 & \\
\hline Grau 1 & $1,19(1,02-1,68)$ & 0,040 & $1,16(0,95-1,78)$ & 0,082 \\
\hline Grau 2 & $1,95(1,77-2,14)$ & 0,020 & $2,10(1,90-2,44)$ & $<0,001$ \\
\hline Não avaliado & $1,12(0,93-1,67)$ & 0,070 & $1,22(0,98-1,64)$ & 0,090 \\
\hline \multicolumn{5}{|l|}{ Episódio reacional } \\
\hline Sem reação & 1 & & 1 & \\
\hline Tipo 1 & $1,32(1,14-1,53)$ & $<0,001$ & $1,50(1,19-1,81)$ & $<0,001$ \\
\hline Tipo 2 & $1,85(1,70-2,01)$ & $<0,001$ & $2,00(1,77-2,31)$ & $<0,001$ \\
\hline Tipos 1 e 2 & $1,17(0,97-1,45)$ & 0,260 & $1,28(0,98-1,42)$ & 0,563 \\
\hline \multicolumn{5}{|c|}{ Número de contatos registrados } \\
\hline$\leq 5$ & 1 & & - & - \\
\hline$>5$ & $0,99(0,92-1,08)$ & 0,990 & - & - \\
\hline \multicolumn{5}{|c|}{ Número de contatos examinados } \\
\hline$\leq 5$ & 1 & & - & - \\
\hline$>5$ & $1,09(0,99-1,19)$ & 0,077 & - & - \\
\hline \multicolumn{5}{|l|}{ Zona de residência } \\
\hline Rural & 1 & & - & - \\
\hline Urbana & $1,85(1,74-1,99)$ & 0,012 & $2,00(1,75-2,36)$ & 0,041 \\
\hline
\end{tabular}


Tabela 3. Análise bruta e ajustada das variáveis sociodemográficas e clínico-epidemiológicas associadas às formas clínicas multibacilares, Imperatriz, Maranhão, Brasil, 2008 a 2017.

\begin{tabular}{|c|c|c|c|c|}
\hline \multirow[b]{2}{*}{ Variáveis } & \multicolumn{4}{|c|}{ Classificação operacional multibacilares } \\
\hline & $\begin{array}{l}\text { RP bruta } \\
\text { (IC95\%) }\end{array}$ & Valor de $\mathbf{p}$ & $\begin{array}{c}\text { RP ajustada } \\
\text { (IC95\%) }\end{array}$ & Valor de $\mathrm{p}$ \\
\hline \multicolumn{5}{|l|}{ Tipo de saída } \\
\hline Cura & 1 & & & \\
\hline Transferências & $1,15(0,98-1,39)$ & 0,922 & - & - \\
\hline Óbito & $1,01(0,91-1,22)$ & 0,824 & - & - \\
\hline Abandono & $1,20(0,99-1,49)$ & 0,221 & - & - \\
\hline
\end{tabular}

${ }^{*}$ RP: razão de prevalência; IC95\%: intervalo de confiança de $95 \%$.

Fonte: SINAN Hanseníase, SVS-UGRSI, Imperatriz-Maranhão, 2019.

da prevalência no período entre 2005 e 2015 , com distribuição heterogênea entre as regiões brasileiras, sendo as Regiões Norte, Nordeste e Centro-Oeste responsáveis pela carga endêmica no território nacional e o estado do Maranhão intendente pelos altos índices de prevalência da Região Nordeste.

A grande extensão territorial do país e as desigualdades socioeconômicas entre regiões constituem as razões explicativas para tal heterogeneidade, e as localidades mais desfavorecidas, do ponto de vista socioeconômico, foram consideradas de maior endemicidade. Como fatores positivos para a redução, foram listadas a implementação da PQT e outras medidas preventivas, como exame de contatos e detecção precoce ${ }^{20}$.

A expansão da rede de diagnóstico e do acesso ao tratamento, oportunizada pela descentralização das atividades de controle da hanseníase com ações integradas à APS por meio da ESF em território brasileiro, é essencial para o controle da doença e tem demonstrado sua relevância em cenários do Sul e Sudeste do país, que têm atingido a meta preconizada pela OMS no tocante ao indicador de prevalência da doença ${ }^{19}$.

Na Região Nordeste, estudo que investigou a relação da hanseníase com a cobertura da ESF em municípios da Paraíba indicou que o aumento da cobertura da ESF contribuiu significativamente para o incremento na taxa de detecção da doença, reforçando que serviços baseados na APS são fundamentais para o diagnóstico precoce e o tratamento oportuno ${ }^{21}$.

Em Imperatriz, no ano de 2008, a cobertura populacional estimada da ESF era de 61,59\% e, em 2017 , reduziu-se a $58,43 \%{ }^{22}$, sendo considera- da inferior à média do Brasil, que era de 63,91\% em 2017 e com equipe formada por médico, enfermeiro, técnico ou auxiliar de enfermagem e agentes comunitários de saúde (ACS), podendo haver uma equipe de Saúde Bucal vinculada. Além desse panorama, a rotatividade profissional nas equipes, as ações incipientes de busca ativa em menores de 15 anos e a detecção precoce dos casos têm favorecido a manutenção da cadeia epidemiológica da doença ${ }^{23}$, o que dificulta sua eliminação e, consequentemente, favorece a subnotificação.

Desse modo, emerge a imprescindibilidade de ampliação das ações de diagnóstico e monitoramento da doença em todas as equipes da ESF articuladas ao centro de referência do município, além de considerar melhorias em elementos, como disponibilidade de recursos humanos, capacidade técnica e sensibilidade das equipes e definição de fluxos e processos de trabalho, essenciais para o controle e a vigilância da doença e oportunos para qualificação das práticas profissionais dadas às especificidades do território. Ademais, os profissionais da ESF necessitam aprimorar a qualidade do cuidado oferecido aos usuários e facilitar a orientação de pacientes e familiares no processo de tratamento, recuperação e autocuidado.

Os ACSs são profissionais inseridos na ESF que podem contribuir ativamente para as ações de controle da hanseníase, devido a sua proximidade com a população. Eles são considerados os principais executores da busca ativa de suspeitos dermatológicos na população, uma vez que a visita domiciliar é um instrumento de trabalho que permite a construção de vínculos de confiança 
com as famílias, a observação de seus hábitos, a detecção de problemas, a troca de informações e a transmissão de orientações em saúde ${ }^{24,25}$.

Dessa forma, as orientações realizadas pelos ACSs, durante as visitas domiciliares, podem ser consideradas a principal estratégia para promover e manter a sensibilização da comunidade em relação à hanseníase ${ }^{25}$. A presente investigação, apesar de não ter avaliado o papel dos ACS no contexto de Imperatriz, suscita reflexões sobre a importância da capacitação e a atuação desse profissional no combate à hanseníase. Nessa perspectiva, a busca ativa assume um papel hegemônico por ser um dispositivo que oportuniza a identificação precoce dos casos, bem como seu aumento na comunidade, além de identificar abandono de tratamento por parte dos doentes ${ }^{10,11}$.

Neste estudo, a maioria dos casos notificados era $\mathrm{MB}$, com predominância de dimorfos, similar a resultados de outros estudos ${ }^{26,27}$. Isso demonstra que ainda há elevada circulação do bacilo, o que torna desafiador controlar a hanseníase, sobretudo por questões operacionais, como detecção precoce, tratamento duradouro, exame de contatos e estigma social da doença.

Observou-se associação estatisticamente significante para a variável sexo, com maior ocorrência de formas $\mathrm{MB}$ entre os homens, com quase duas vezes mais ocorrência quando comparados às mulheres. Alguns autores têm apontado que tanto a hanseníase como as formas MB da doença são mais frequentes nos homens do que nas mulheres $^{28,29}$. Esse predomínio provavelmente está vinculado à maior exposição em atividades relacionadas ao trabalho, à pouca procura pelos serviços de saúde, ao baixo nível de autocuidado e ao reduzido acesso a informações ${ }^{28}$.

A baixa adesão do público masculino aos serviços de saúde pode estar relacionada ao horário de funcionamento das unidades, o qual pode coincidir com o exercício das atividades laborais ${ }^{9}$, o que dificulta o acesso e a permanência do sexo masculino nesse nível de atenção $0^{30}$.

Estudos apontaram que as mulheres tendem a frequentar mais regularmente os serviços de saúde e possuem maior preocupação com hábitos saudáveis ${ }^{31,32}$, o que também deve ser considerado na diferença quanto ao sexo. Nesse sentido, o sexo deve ser reconhecido como fator determinante da ocorrência e da maior gravidade da doença ${ }^{28}$, o que aponta para a inevitabilidade de estratégias diferenciadas voltadas ao público masculino, contemplando a diversidade do panorama da hanseníase no país. A ampliação do horário de atendimento pelos profissionais da ESF no cenário sob investigação poderia ser alternativa estratégica para atendimento desse público-alvo.

Em relação à idade, observou-se maior acometimento na faixa etária entre 30 e 59 anos $(\mathrm{RP}=1,60)$ e em idade $\geq 60$ anos $(\mathrm{RP}=1,72)$, associando-se estatisticamente com a forma clínica MB. Conforme a idade avança, maior é a possibilidade de contrair as formas mais bacilíferas, semelhante aos resultados encontrados em outros estudos $^{33,34}$. Esses achados também evidenciaram o predomínio da hanseníase na faixa etária economicamente ativa ${ }^{29}$ e configuram-se como de grande relevância, pois a fase produtiva pode ser drasticamente interrompida, devido ao alto poder incapacitante da doença.

A baixa escolaridade teve associação significativa com as formas $\mathrm{MB}$, na qual observou-se que indivíduos com escolaridade $<8$ anos estiveram mais suscetíveis $(\mathrm{RP}=2,5)$, concordando com resultado encontrado por Sousa et al..$^{35}$. Outros estudos têm revelado predominância do nível baixo de instrução com os casos de hanseníase $^{36,37}$.

Um aspecto preocupante da baixa escolaridade relaciona-se ao fato de se tratar de risco para o surgimento de incapacidades físicas, pois o aumento do nível de escolaridade dos indivíduos tende a diminuir a frequência de diagnósticos com incapacidades físicas instaladas, e pessoas analfabetas e com ensino fundamental possuem mais chances de serem diagnosticadas com incapacidades em comparação àquelas com ensino médio ou mais ${ }^{38}$. Nessa linha de raciocínio, a relação entre escolaridade e hanseníase pode ser explicada pela menor compreensão de sinais e sintomas da doença, dificultando a adoção do tratamento e medidas para o autocuidado, além da dificuldade do acesso aos serviços de saúde que essa população está sujeita ${ }^{39,40}$.

Destarte, a educação em saúde realizada pela ESF apresenta-se como importante fator de determinação da saúde ${ }^{25,39}$, deixando explícita a primordialidade de ampliação de investimentos públicos em educação, além da atenuação das desigualdades socioeconômicas, que podem incrementar o controle da hanseníase, bem como melhorar as condições de vida da população.

Neste estudo, o grau 2 de incapacidade física teve associação significativa com as formas clínicas $\mathrm{MB}$, sugerindo diagnóstico tardio da hanseníase, similar aos dados de outros estudos ${ }^{26,41-43}$. A deficiência, a incapacidade e a deformidade são as principais causas do estigma associado à 
doença e interferem sobremaneira na qualidade de vida dos portadores de hanseníase ${ }^{44}$. Ademais, Leano et al. ${ }^{40}$ destacam que a gravidade da hanseníase se mostra nas incapacidades físicas provenientes dos casos não tratados, o que traz redução ou eliminação das oportunidades de trabalho e de subsistência.

As evidências apontadas em Imperatriz remetem à discussão sobre o cumprimento das metas da Estratégia Global para Hanseníase 2016-2020 pactuadas pela OMS, cujo princípio-chave era a detecção precoce de todos os pacientes antes do surgimento de incapacidades ${ }^{45}$. Considerando que a detecção precoce e a redução de incapacidades parecem estar relacionadas à eficiência dos serviços da APS ${ }^{19,20}$, ressalta-se a importância de melhorar a oferta dos serviços de saúde, no que diz respeito ao acesso, à aplicação de recursos e à capacitação profissional. Desse modo, faz-se necessária a capacitação periódica direcionada às necessidades dos profissionais que atuam na ESF do município de Imperatriz, visando à oferta de uma assistência mais qualificada, no tocante à detecção precoce dos sinais e sintomas da hanseníase.

Quanto à presença de episódios reacionais $1 \mathrm{e}$ 2 , identificou-se associação estatisticamente significativa dessa variável com as formas $\mathrm{MB}$, com $\mathrm{RP}$ respectivas de 1,50 e 2,00. Resultado similar foi encontrado por Monteiro et al. ${ }^{46} \mathrm{em}$ estudo realizado em Tocantins, os quais reconhecem que os casos MB representam fatores de risco para o desenvolvimento de episódios reacionais, mais frequentes nos casos MB.

Correlacionar as formas clínicas e os estados reacionais é extremamente importante, pois esses episódios constituem intercorrências no curso da doença e são as principais causas de incapacidades nesses pacientes ${ }^{4,47}$. Para evitar as complicações clínicas decorrentes dessas intercorrências, é necessário reforçar a importância da prevenção, aliada à PQT específica, a qual proporciona novas perspectivas de cura para a hanseníase ${ }^{47}$, sem necessidade de reiniciá-la, bem como não a contraindicam ${ }^{4}$.

No que diz respeito à área de residência, a zona urbana apresentou significância estatística com as formas clínicas MB. Os moradores desses espaços tiveram duas vezes mais chances de adoecer quando comparados aos residentes da zona rural, resultados que coadunam com outros estudos ${ }^{48,49}$, nos quais os casos de hanseníase foram identificados essencialmente nos cenários urbanos.

O processo de urbanização da hanseníase no Brasil intensificou-se nas últimas décadas, prin- cipalmente devido a condições precárias de vida da população e ao acesso restrito aos bens e serviços coletivos. Essas características relacionam-se no espaço urbano fragilizado diante da elevada densidade populacional e da vulnerabilidade socioeconômica, que determinam, em conjunto, um panorama geral de adoecimento e morte $\mathrm{e}^{50}$. Entretanto, é importante destacar que o reduzido número de casos notificados na zona rural do cenário sob investigação pode estar relacionado a desigualdades no acesso à saúde, a menor oferta de serviços de saúde nessas áreas e a menor procura por esses serviços pela população rural, quando comparados à população da zona urba$\mathrm{na}^{51}$. Esses fatores podem resultar em número de notificações abaixo do real valor existente.

Um achado que merece ser destacado neste estudo é o grande número de respostas ignoradas em informações importantes obtidas a partir de campos de preenchimento considerados essenciais e obrigatórios, o que significa a existência de falhas na notificação da doença e/ou alimentação indevida, dificultando o rastreio e o direcionamento da assistência adequada ao portador da doença. A alimentação dos sistemas de informação depende diretamente do empenho dos municípios e regiões em fornecer os dados ${ }^{34}$, bem como do adequado preenchimento da ficha de notificação por parte dos profissionais da ESF envolvidos na atenção aos pacientes.

Entre as limitações deste estudo, destaca-se a utilização de dados secundários que, muitas vezes, apresentam ausência de informações e inconsistência dos dados. Portanto, faz-se necessário o preenchimento adequado das fichas de notificação compulsória, com informações consistentes, para que estes dados forneçam subsídios para a avaliação do estado de saúde da população e tomada de decisões.

\section{Conclusão}

A maioria dos pacientes era portadora da forma clínica multibacilar, com maior acometimento da população economicamente ativa e baixo nível de instrução. Níveis alto e muito alto de endemicidade foram evidenciados, o que demonstra que ainda existem falhas, por parte da gestão e dos serviços de saúde, no controle dessa enfermidade, desde ações de prevenção, manejo e cura.

Tais achados suscitam a necessidade de fortalecer as ações de vigilância em saúde, sobretudo pelos profissionais da Estratégia Saúde da Família articulados ao centro de referência do muni- 
cípio de Imperatriz com foco na busca ativa e no tratamento precoce, com vistas à redução de incapacidades físicas e do consequente prejuízo na qualidade de vida dos portadores desse agravo.

\section{Colaboradores}

FC Lopes e M Santos Neto participaram da concepção do estudo, análise e interpretação dos dados, redação, revisão do artigo e aprovação da versão final. ACV Ramos, LM Pascoal, FS Santos, ILTP Rolim, MAAO Serra e LH Santos colaboraram na análise e interpretação dos dados, revisão do artigo e aprovação da versão final.

\section{Agradecimentos}

Pesquisa financiada pela Fundação de Amparo à Pesquisa e ao Desenvolvimento Científico e Tecnológico do Maranhão (FAPEMA), processo UNIVERSAL (01036/19), e pela Coordenação de Aperfeiçoamento de Pessoal de Nível Superior Brasil (CAPES), através do código 001. Agradecemos ainda ao Serviço de Vigilância em Saúde da Unidade Gestora Regional de Saúde de Imperatriz pela concessão dos dados.

\section{Referências}

1. World Health Organization (WHO). Guidelines for the diagnosis, treatment and prevention of leprosy [Internet]. Geneva: WHO; 2018 [cited $2021 \mathrm{Feb}$ 16]. Available from: https://apps.who.int/iris/handle/10665/274127

2. Rodrigues RN, Leano HA, Bueno IC, Araújo KM, Lana FC. Áreas de alto risco de hanseníase no Brasil, período 2001-2015. Rev Bras Enferm 2020; 73(3): e20180583.

3. Souza CD, Magalhães MA, Luna CF. Hanseníase e carência social: definição de áreas prioritárias em estado endêmico do Nordeste brasileiro. Rev Bras Epidemiol 2020; 3: e200007.

4. Brasil. Ministério da Saúde (MS). Guia prático sobre a hanseníase [Internet]. Brasília: MS; 2017 [acessado 2021 fev 16]. Disponível em: https://portalarquivos2. saude.gov.br/images/pdf/2017/novembro/22/Guia -Pratico-de-Hanseniase-WEB.pdf

5. Barbosa CC, Bonfim CV, Brito CM, Ferreira AT, Gregório VR, Oliveira AL, Portugal JL, Medeiros ZM. Spatial analysis of reported new cases and local risk of leprosy in hyper-endemic situation in Northeastern Brazil. Trop Med Int Health 2018; 23: 748-757. 
6. Mitjà $\mathrm{O}$, Marks M, Bertran L, Kollie K, Argaw D, Fahal AH, Fitzpatrick C, Fuller LC, Isquierdo BG, Hay R, Ishii N, Johnson C, Lazarus JV, Meka A, Murdoch M, Ohene SA, Small P, Steer A, Tabah EN, Tiendrebeogo A, Waller L, Yotsu R, Walker SL, Asiedu K. Integrated Control and Management of Neglected Tropical Skin Diseases. PLoS Negl Trop Dis [Internet]. 2017 [acess 2021 Feb 16]. Available from: https://journals. plos.org/plosntds/article?id=10.1371/journal.pntd. 0005136

7. World Health Organization (WHO). Global leprosy update, 2018: moving towards a leprosy free world. Wkly Epidemiol Rec [Internet]. 2019 [cited $2021 \mathrm{Feb}$ 16]; 94(35;36): 389. Available from: https://apps.who. int/iris/handle/10665/326776

8. Brasil. Ministério da Saúde (MS). Departamento de Condições Crônicas e Infecções Sexualmente Transmissíveis. Boletim epidemiológico de hanseníase [Internet]. Brasília: MS; 2020 [acessado 2021 fev 17]. Disponível em: http://www.aids.gov.br/pt-br/pub/2020/ boletim-epidemiologico-de-hanseniase-2020

9. Serra MA, Santos CS, Lima Neto PM, Oliveira KG, Oliveira FJ, Gordon AS, Matos DP, Lima RJCP, Bezerra JM, Dias ICCM, Santos FS, Costa ACPJ, Santos Neto M, Silva, AR. Araújo MFM. Factors associated with multibacillary leprosy in a priority region for disease control in Northeastern Brazil: a retrospective observational study. J Trop Med [Internet] 2019 [cited 2021 Feb 16]. Available from: https://www.mendeley.com/ catalogue/18bedf85-c42f-3ece-b740-119392049bd9/

10. Savassi LCM, Modena CM. Hanseníase e a atenção primária: desafios educacionais e assistenciais na perspectiva de médicos residentes. Hansen Int 2015; 40(2): 2-16.

11. Neta OAG, Arruda GMMS, Carvalho MMB, Gadelha RRM. Percepção dos profissionais de saúde e gestores sobre a atenção em hanseníase na Estratégia Saúde da Família. Rev Bras Promoc Saúde 2017; 30(2):239-248.

12. Espíndola MF, Nascimento JL, Gomes AC, Costa AL, Garcia FN, Rodrigues MC, Regis GJS, Vilas Boas, GF, Arruda EL. Perfil epidemiológico da hanseníase no período de 2015 a 2018 no município de Goianésia (GO). Br J Health Rev 2020; 3(2): 2600-2611.

13. Rouquayrol MZ, Silva MG. Rouquayrol epidemiologia \& saúde. 8a ed. Rio de Janeiro: MedBook; 2018.

14. Instituto Brasileiro de Geografia e Estatística (IBGE). População - Imperatriz, MA [Internet]. Brasília: IBGE; 2018 [acessado $2021 \mathrm{fev} 16$ ]. Disponível em: https:// cidades.ibge.gov.br/brasil/ma/imperatriz/panorama

15. Santos LS, Nunes FG. Imperatriz do Maranhão: proposição para a compreensão do processo de ocupação e consolidação da cidade. GeoTextos 2018; 14(2): 117-141.

16. Brasil. Ministério da Saúde (MS). Diretrizes para vigilância, atenção e eliminação da hanseníase como problema de saúde pública [Internet]. Brasília: MS; 2016 [acessado 2021 fev 17]. Disponível em: https://portalarquivos2.saude.gov.br/images/pdf/2016/fevereiro/04/diretrizes-eliminacao-hanseniase-4fev16-web. pdf

17. Barros AJ, Hirakata VN. Alternatives for logistic regression in crosssectional studies: an empirical comparison of models that directly estimate the prevalence ratio. BMC Med Res Methodol 2003; 3:21.
18. World Health Organization (WHO). Global leprosy strategy 2016-2020: accelerating towards a leprosy-free world [Internet]. New Delhi: WHO; 2017 [cited 2021 Feb 16]. Available from: https://apps.who.int/iris/handle/ 10665/254907

19. Brasil. Ministério da Saúde (MS). Estratégia nacional para o enfrentamento da hanseníase (2019-2022) [Internet]. Brasília: MS; 2019 [acessado 2021 fev 16]. Disponível em: http://portalarquivos2.saude.gov. br/images/pdf/2019/marco/27/Estrategia-NacionalCGHDE-Consulta-Publica-27mar.pdf

20. Ribeiro MD, Silva JC, Oliveira SB. Estudo epidemiológico da hanseníase no Brasil: reflexão sobre as metas de eliminação. Rev Panam Salud Publica 2018; 42:e42.

21. Araújo KMFFA, Lana FCF. Relação da hanseníase com a cobertura da estratégia saúde da família e condições socioeconómicas. Ciencia y Enfermeria 2020; 26(1): $1-9$.

22. Brasil. Ministério da Saúde (MS). Cobertura da Atenção Básica [Internet]. Brasília: MS; 2020 [acessado 2021 fev 16]. Disponível em: https://egestorab.saude. gov.br/paginas/acessoPublico/relatorios/relHistoricoCoberturaAB.xhtml

23. Gordon AS, Gomes JM, Costa AC, Serra MA, Santos Neto M, Xavier MB. Incidência de hanseníase em menores de 15 anos acompanhados no município de Imperatriz, Maranhão, entre 2004 e 2010. Arq Cienc Saúde Unipar 2017; 21(1):19-24.

24. Vieira NF, Lanza FM, Martínez-Riera JR, Nolasco A, Lana FCF. Orientación de la atención primaria en las acciones contra la lepra: factores relacionados con los profesionales. Gaceta Sanitaria 2020; 34:120-126.

25. Lanza FM, Lana FCF. O processo de trabalho em hanseníase: tecnologias e atuação da equipe de saúde da família. Texto Contexto Enferm 2011; 20(Esp):238-246.

26. Mendes AO, Costa CE, Silva RC, Campos SA, Cunha VM, Silva GC, Silva MCS. Caráter clínico-epidemiológico e grau de incapacidade física nos portadores de hanseníase no município de Barbacena - MG e macrorregião no período de 2001 a 2010. Rev Med Minas Gerais 2014; 24(4):486-494.

27. Reis MC, Raposo MT, Alencar CH, Ramos Júnior AN, Heukelbach J. Incapacidades físicas em pessoas que concluíram a poliquimioterapia para hanseníase em Vitória da Conquista, Bahia, Brasil. Acta Fisiatr 2018; 25(2):78-85.

28. Souza EA, Ferreira AF, Boigny RN, Alencar $\mathrm{CH}$ Heukelbach J, Martins-Melo FR, Barbosa JC, Ramos Jr AN. Hanseníase e gênero no Brasil: tendências em área endêmica da região Nordeste, 2001-2014. Rev Saude Publica 2018; 52:20.

29. Ramos AC, Yamamura M, Arroyo LH, Popolin MP, Chiaravalloti Neto F, Palha PF, Uchoa SAC, Pieri FM, Pinto IC, Fiorati RC, Queiroz AAR, Belchior AS, Santos DT, Garcia MCCG, Crispim, JA, Alves, LS, Berra TZ, Arcêncio RA. Spatial clustering and local risk of leprosy in São Paulo, Brazil. PLoS Negl Trop Dis [Internet]. 2017 [cited $2021 \mathrm{Feb}$ 16]. Available from: https:// repositorio.usp.br/item/002879810

30. Moreira RL, Fontes WD, Barboza TM. Dificuldades de inserção do homem na atenção básica a saúde: a fala dos enfermeiros. Esc Anna Nery 2014; 18(4): 615-621. 
31. Levorato CD, Mello LM, Silva AS, Nunes AA. Fatores associados à procura por serviços de saúde numa perspectiva relacional de gênero. Cienc Saude Colet 2014; 19(4): 1263-1274.

32. Thompson AE, Anisimowicz Y, Miedema B, Hogg W, Wodchis WP, Aubrey-Bassler K. The influence of gender and other patient characteristics on health careseeking behaviour: a Qualicopc Study. BMC Family Practice 2016; 17:38.

33. Monteiro MJ, Santos GM, Barreto MT, Silva RV, Jesus RL, Silva HJ. Perfil epidemiológico de casos de hanseníase em um estado do Nordeste Brasileiro. Rev Aten Saude 2017; 15(54):21-28.

34. Oliveira AK, Freire FF, Nascimento MR. Incidência e perfil clínico-epidemiológico da hanseníase no Brasil. Revista Científica da Fasete [Internet]. 2018 [acessado 2021 Feb 16]. Disponível em: https://www.unirios. edu.br/revistarios/media/revistas/2018/20/incidencia_e_perfil_clinico_epidemiologico_da_hanseniase_no_brasil.pdf

35. Sousa GC, Vieira FS, Oliveira DE, Costa ES, Moura ME. Caracterização clínico-epidemiológica de casos de hanseníase com incapacidades físicas. Rev Rene 2017; 18(1): 99-105.

36. Basso ME, Silva RL. Perfil clínico-epidemiológico de pacientes acometidos pela hanseníase atendidos em uma unidade de referência. Rev Soc Bras Clin Med 2017; 15(1): 27-32.

37. Boigny RN, Souza EM, Romanholo HS, Araújo OD, Araújo TM, Carneiro MAG, Grijó MDF, Henz NLFB, Reis AS, Pinto, MSAP, Barbosa JC, Ramos Jr AN. Persistência da hanseníase em redes de convívio domiciliar: sobreposição de casos e vulnerabilidade em regiões endêmicas no Brasil. Cad Saude Publica 2019; 35(2):e00105318.

38. Lages DS, Kerr BM, Bueno IC, Niitsuma EN, Lana FC. A baixa escolaridade está associada ao aumento de incapacidades físicas no diagnóstico de hanseníase no Vale do Jequitinhonha. HU Revista 2018; 44(3): 303-309.

39. Souza DE. Determinação social da saúde: associação entre sexo, escolaridade e saúde autorreferida [tese]. Salvador: Instituto de Saúde Coletiva da UFBA; 2012.

40. Leano HA, Araújo KM, Bueno IC, Niitsuma EN, Lana FC. Fatores socioeconômicos relacionados à hanseníase: revisão integrativa da literatura. Rev Bras Enferm 2019; 72(5): 1474-1485.

41. Araújo EJ, Araújo OD, Araújo TM, Almeida PD, Sena IV, Neri EA. Pós-alta de hanseníase: prevalência de incapacidades físicas e sobreposição de doenças. Cogitare Enferm 2018; 23(4): e58080.

42. Darlong J, Govindharaj P, Darlong F, Mahato N. A study of untreated leprosy affected children reporting with Grade 2 disability at a referral centre in West Bengal, India. Lepr Rev 2017; 88: 298-305.

43. Portela NL, Sousa PH, Melo LN. Fatores associados à incapacidade física de casos novos de hanseníase em Paço do Lumiar - MA, 2006-2015. Hygeia 2018; 14(27): 80-88.
44. Reyila VP, Betsy A, Riyaz N, Sasidharanpillai S, Sherjeena PV, Majitha MP, Joseph DM. Clinico-epidemiological study of disability due to leprosy at the time of diagnosis among patients attending a tertiary care institution. Indian J Dermatol. 2019; 64(2):106-111.

45. World Health Organization (WHO). Global leprosy update, 2016: accelerating reduction of disease burden [Internet]. Geneva: WHO; 2017 [cited 2021 Feb 16]. Available from: https://apps.who.int/iris/bitstream/ handle/10665/258841/WER9235.pdf? sequence $=1$

46. Monteiro LD, Alencar CH, Barbosa JC, Braga KP, Castro $\mathrm{MD}$, Heukelbach J. Incapacidades físicas em pessoas acometidas pela hanseníase no período pós-alta da poliquimioterapia em um município no Norte do Brasil. Cad Saude Publica 2013; 29(5): 909-920.

47. Coelho Júnior LG, Machado GB, Faria TA. Reação hansênica tipo dois em paciente multibacilar, forma Virchowiana, em vigência de tratamento: relato de caso. Rev Med (São Paulo) 2015; 94(3): 197-200.

48. Santos DA, Spessatto LB, Melo LS, Olinda RA, Lisboa HC, Silva MS. Prevalência de casos de hanseníase. Rev Enferm UFPE Online 2017; 11(Supl. 10):4045-4055.

49. Gonçalves NV, Alcântara RC, Sousa Júnior AS, Pereira AL, Miranda CS, Oliveira JSS, Melo ACBV, Guedes JA, Costa RJF, Costa SBN, Marcos W, Gomes RP, Oliveira RAC, Palácios VRCM. A hanseníase em um distrito administrativo de Belém, estado do Pará, Brasil: relações entre território, socioeconomia e política pública em saúde, 2007-2013. Rev Pan-Amaz Saude 2018; 9(2): 21-30.

50. Barbosa DR, Almeida MG, Santos AG. Características epidemiológicas e espaciais da hanseníase no Estado do Maranhão, Brasil, 2001-2012. Med (Ribeirão Preto) 2014; 47(4): 347-356.

51. Arruda NM, Maia AG, Alves LC. Desigualdade no acesso à saúde entre as áreas urbanas e rurais do Brasil: uma decomposição de fatores entre 1998 a 2008. Cad Saude Publica 2018; 34(6): e00213816.

Artigo apresentado em 03/08/2020

Aprovado em 18/02/2021

Versão final apresentada em 20/02/2021

Editores-chefes: Romeu Gomes, Antônio Augusto Moura da Silva 